\title{
"El que vende tierra, come tierra": cambios y continuidades en las haciendas de la cuenca del río Amaime, Valle del Cauca, Colombia, siglo XIX
}

\section{"EL QUE VENDE TIERRA COME TIERRA"1: CHANGES AND CONTINUITIES IN ESTATES IN THE AMAIME RIVER BASIN, VALLE DEL CAUCA, COLOMBIA, 19TH CENTURY}

\author{
"QUEM VENDE TERRA COME TERRA"2: MUDANC AS E CONTINUIDADES \\ NAS FAZENDAS DA BACIA DO RIO AMAIME, VALLE DEL CAUCA, \\ COLÔMBIA, SÉCULO XIX
}

Para citar este artículo: Giraldo Hoyos, M. (2019). "El que vende tierra, come tierra": cambios y continuidades en las haciendas de la cuenca del río Amaime, Valle del Cauca, Colombia, siglo XIX.

Perspectiva Geográfica, 24(2), 13-34. doi: 10.19053/01233769.10137

Martín Giraldo-Hoyos ${ }^{3}$

Recepción:

20 de junio de 2019

Evaluación:

9 de septiembre de 2019

Aprobación:

20 de septiembre de 2019

\section{Resumen}

Este artículo explora las dinámicas de tenencia de la tierra y uso del suelo de las haciendas en el valle del río Cauca durante la segunda mitad del siglo XIX, con base en casos ubicados en la cuenca del río Amaime, tributario del Cauca. Con la

$1 \quad$ Literally, "he who sells his land ends up eating dirt." This local saying means that a person who sells their estate is bound to a life of poverty.

2 Esta giria quer significar que uma pessoa que vende seu terreno vincula-se para uma vida de pobreza

3 Historiador de la Universidad Nacional de Colombia, sede Bogotá, con Maestría en Historia de Universidad de Saskatchewan, Canadá. Actualmente es estudiante de doctorado en la Universidad de McGill, Canadá. Este artículo contiene un fragmento de la tesis de maestría del autor, titulada Owning Land, Appropriating Nature. The Configuration of an Agricultural Landscape in the Cauca River Valley, South Western Colombia, 1864-1901, elaborada en el marco del proyecto de investigación internacional "Sustainable Farm Systems: long term socioecological metabolism in western agriculture". https://orcid.org/0000-0002-1977-5119. jmgiraldoh@unal.edu.co. 
intención de complejizar la narrativa de estancamiento productivo y espacial creada sobre esta región, acá se analizan las dinámicas de transformación espacial en determinadas haciendas, evidenciando así su sistemática mutación espacial y productiva durante el proceso histórico de liberalismo decimonónico. Para esto se consultaron diferentes fuentes documentales, incluyendo correspondencia, cartografía histórica y documentos notariales. El uso de un sistema de información geográfico histórico permitió georreferenciar datos notariales para el análisis y la visualización espacial de cambios en la tenencia de la tierra dentro de la ventana de estudio. Los casos de las haciendas La Concepción y La Torre demuestran, desde trayectorias diferentes, que a partir de la década de 1850 se dieron procesos significativos de reorientación productiva y diversificación social en el Valle.

Palabras clave: agricultura, geografía histórica, historia latinoamericana, liberalismo, tenencia de la tierra.

\section{Abstract}

This article explores the dynamics of land tenure and use in the Cauca river valley during the second half of the 19th century, focusing on estates in the Amaime river basin - a tributary of the Cauca River-. In order to elaborate on the narrative about the alleged productive and spatial stagnation of this region, spatial transformation of certain estates are discussed, thus showing their systematic spatial and productive mutation during the historical process of 19th-century liberalism. For this, various documentary sources were searched, including correspondence, historical cartography and notary documents. A historical geographic information system was used to georeference notary data for the spatial analysis and visualization of land tenure changes within the study period. From different track records, the cases of La Concepción and La Torre estates prove that significant processes of productive reorientation and social diversification have taken place in the valley since the 1850s.

Keywords: Agriculture, historical geography, Latin American history, liberalism, land tenure.

\section{Resumo}

O presente artigo explora as dinâmicas da posse da terra e uso do solo nas fazendas no vale do Rio Cauca durante a segunda metade do século XIX, com base em casos localizados na bacia do rio Amaime, afluente de Cauca. Com a intencionalidade 
de complexificar a narrativa de estagnação produtiva e espacial criada sobre esta região, aqui é analisada a dinâmica da transformação espacial em certas fazendas, demonstrando desse jeito sua sistemática mutação espacial e produtiva durante o processo histórico do liberalismo do século XIX. Para isso, foram consultadas diferentes fontes documentais, incluindo correspondência, cartografia histórica e documentos notariais. $O$ uso de um sistema de um sistema de informações geográficas históricas permitiu georreferenciar dados notariais para a análise e a visualização espacial de mudanças na posse da terra dentro da janela do estudo. Os casos das fazendas La Concepción e La Torre demonstram, a partir de diferentes trajetórias, que desde a década de 1850 deram-se processos significativos de reorientação produtiva e diversificação social no Vale.

Palavras chave: agricultura, geografia histórica, história da América Latina, liberalismo, posse da terra.

\section{Introducción}

Tras terminar otra jornada de viaje por el valle del río Cauca en 1857, el naturalista norteamericano Isaac Holton (1857) meditaba: "otro día ha pasado sin más cambio en el Valle del Cauca que en la faz del océano, y así han pasado generaciones" (p. 467, traducción propia). Al igual que Holton, otros extranjeros destacaron en sus relatos de viaje la sensación de monotonía que les despertaba este paisaje agropecuario "donde la agricultura está reducida casi por completo al sistema pastoril”, en palabras del botánico francés Edouard André (2013) en 1875 (p. 17) ${ }^{4}$. Sustentada sobre la idealización de la riqueza natural de estas planicies aluviales como espacio privilegiado para la agricultura tropical, esta percepción de estatismo

$4 \quad$ Como lo explica la historiadora Katherinne Mora para el caso de la sabana de Bogotá, la idea de monotonía constituye un patrón recurrente en los relatos de viajeros debido a su inclinación por hacer juicios generalizadores con base en visitas fugaces (Mora Pacheco, 2015). productivo sirvió de base para proyectos modernizadores en diferentes épocas. Mientras que en el siglo XIX esta fue la narrativa de los liberales para fomentar proyectos exportadores, una visión semejante serviría a tecnócratas y científicos para respaldar el proyecto agroindustrial azucarero en el siglo XX.

En el marco de la modernización agrícola en la década de 1950, la investigación en geografía humana del estadounidense Raymond Crist (1952) enfatizó en el dominio histórico de la hacienda colonial como causa esencial del estancamiento económico y social en la región. Este modelo, en sus palabras, "no era conducente a la intensificación de la agricultura en las tierras más fértiles para el bienestar económico de las mayorías, por el contrario, condujo a la invasión del pastoreo sobre cultivos" (p. 35, traducción propia). Para Crist, la paradoja del sistema de tenencia de la tierra en el Valle se explicaba porque la clase terrateniente ha privilegiado históricamente el significado de 
la tierra como fuente de prestigio por encima de la productividad. El fenómeno denominado como "tenencia de prestigio" sería uno de los ejes de análisis de la historiografía latinoamericana para explicar las lógicas de la hacienda colonial a través del tiempo (Mörner, 1973, p. 193), así como para exponer las continuidades coloniales en el mundo agrario como lastres de desigualdad social y atraso económico.

Evidente fuente de desigualdad territorial en la historia de Latinoamérica, numerosos estudios han demostrado que el monopolio de la tierra como fuente de prestigio social y poder político por parte de élites terratenientes se ha perpetuado por medio de la ganadería y el uso del pastoreo como forma de expandir y retener el control sobre la tierra (Reyes Posada, 1978; Guillén, 1979; Hecht, 1993). No obstante, de acuerdo al historiador ambiental Shawn Van Ausdal, estas lecturas de la ganadería como un medio más que como un fin han desdeñado análisis rigurosos sobre las lógicas internas de las haciendas ganaderas en la historia de Colombia y desconocen problemáticas como la expansión de pastos y la deforestación (Van Ausdal, 2009a, p. 3). Entender las dinámicas sociales, económicas y ambientales de las haciendas en el marco de sus respectivos contextos históricos y geográficos permite abarcar los roles de los diferentes actores involucrados en procesos agrarios con repercusiones significativas en la actualidad. Siguiendo esta idea, la presente investigación busca cuestionar la noción de estancamiento productivo y espacial en las haciendas del valle geográfico del río Cauca en la segunda mitad del siglo XIX, para lo cual se analizan las dinámicas de transformación espacial en determinados sectores de la cuenca del río Amaime, tributario del Cauca entre los actuales municipios de El Cerrito y Palmira. Lejos de está- ticas y homogéneas, las haciendas de esta parte del Valle sufrieron cambios estructurales que resultan significativos para explicar la transición socioecológica de la agricultura sufrida en la región durante el siglo XX (Marull, Delgadillo, Cattaneo, La Rota \& Krausmann, 2018).

Este artículo explora las dinámicas históricas de tenencia de la tierra y uso del suelo en el valle del río Cauca en la segunda mitad del siglo XIX, con base en los casos de las haciendas La Concepción y La Torre en la cuenca del río Amaime. Desde la perspectiva de la historia agraria y por medio de un ejercicio con un sistema de información geográfico histórico (SIG-H), esta investigación busca revisitar la interpretación tradicional sobre el estatismo del régimen agrario en el Valle y explora la sistemática mutación espacial y productiva de las haciendas en las riberas del Amaime al compás de los procesos históricos experimentados en la región durante el auge y el declive del liberalismo decimonónico. La configuración del paisaje agropecuario en el valle geográfico del siglo XIX fue resultado del encuentro entre diversos actores atraídos por la disponibilidad de tierras fértiles, pero con variadas formas de apropiarse, de utilizar y de transformar los privilegiados recursos naturales de este valle.

En la segunda mitad del siglo XIX las tierras del valle del río Cauca fueron objeto de un agitado mercado inmobiliario, cuando las haciendas señoriales, establecidas allí bajo los términos del orden colonial, enfrentaron el impacto del reformismo liberal republicano. La independencia y las guerras civiles que le siguieron provocaron la desarticulación de los mercados mineros y los circuitos comerciales sobre los que se sostenía la economía regional con epicentro en Cali, y de los cuales de- 
pendía el mundo agropecuario de las planicies al otro lado del río Cauca (Colmenares, 1975; Escorcia, 1982). Sin bases financieras para sostener sus vidas urbanas, las élites terratenientes y mineras volcaron sus expectativas sobre las haciendas patrimoniales, cuyas tierras podían ser libremente comercializadas desde la extinción de los derechos de mayorazgo proclamada en la Constitución de 1824. En su afán por promover el libre mercado de tierras, el usufructo de tierras "incultas" y atraer inversión extranjera, las políticas liberales, además del mayorazgo, eliminaron la figura de resguardos indígenas en 1863 —restituida en 1890-, ejecutaron la desamortización de bienes eclesiásticos en 1861 y adjudicaron tierras baldías en diferentes momentos. En consecuencia, empresarios nacionales y extranjeros pusieron su mirada sobre el valle geográfico, cuyas fértiles tierras ofrecían un gran potencial para empresas agropecuarias, además de contar con una ubicación geográfica estratégica dentro del circuito comercial del océano Pacífico.

Adicionalmente, las dinámicas de colonización interna desde el siglo XVIII se acentuaron en las cordilleras Central y Occidental para mediados del siglo XIX (LeGrand, 1988). En el Valle, la abolición de la esclavitud en 1851 permitió a numerosas familias previamente vinculadas al circuito existente entre haciendas esclavistas y minas en la costa pacífica establecer comunidades en espacios marginados por el orden territorial colonial como selvas, piedemontes, zonas inundables y cruces de caminos (Mina, 1975; Mejía Parado, 1993). A las comunidades afrodescendientes se sumaron también campesinos que desde la región antioqueña buscaron opciones de asentamiento, principalmente al norte de la zona plana y en piedemontes circundantes (Almario García, 2013). En conse- cuencia, durante este periodo la apretada red de latifundios distribuidos a lo largo del valle geográfico sufrió la intensa presión de nuevos y viejos actores en la región, unos con necesidades de subsistencia, pero sin capacidad adquisitiva, otros con los medios e intereses productivos del liberalismo decimonónico.

La perspectiva espacial ha sido cardinal en la historiografía sobre la configuración histórica del Valle del Cauca. El particular devenir del sector agropecuario en esta región ha inspirado numerosas inquietudes científicas pertinentes para entender la consolidación del Valle como región independiente del Gran Cauca, así como su trayecto hacia la formación del clúster agroindustrial y del monocultivo de caña de azúcar, con consecuencias sociales y ambientales asociadas a tal monopolio productivo. Así, desde la década de 1950, historiadores y geógrafos han encontrado, en metodologías de análisis espacial y herramientas cartográficas, plataformas útiles para estudiar las dinámicas socioeconómicas (Colmenares, 1975; Valdivia Rojas, 1992; Almario García, 2013), agrarias (Crist, 1952; Mejía Parado, 1993; Llanos Vargas, 1979), y ambientales (Velasco Arizabaleta, 1982; Motta González \& Perafán Cabrera, 2012; Perafán Cabrera, 2012; Delgadillo Vargas, 2014) de este proceso histórico.

Aunque el dominio de enfoques espaciales para explicar procesos históricos no es excepcional en el contexto colombiano (Montañez Gómez, 1999), en el que historia y geografía han operado en constante diálogo, en el Valle este patrón resulta especialmente evidente. No es coincidencia que en 1979 hayan nombrado Historia y Espacio a la revista de historia de la Universidad del Valle, en parte por la influencia del geógrafo Luis Valdivia Rojas. Desde la década de 1970, Valdivia llevó a 
cabo ejercicios pioneros de análisis espacial sobre procesos históricos en Colombia y el Valle, que incluyen análisis de cartografía histórica, georreferenciación de datos demográficos del siglo XIX y rigurosos estudios espaciales de procesos sociales y económicos (Valdivia Rojas, 1979; 1980; 1984; 1992). Inspirado por el legado de este autor y en la línea espacial de la historiografía vallecaucana, la investigación de la que a continuación se presenta un fragmento se llevó a cabo haciendo uso de metodologías SIG desde una perspectiva histórica.

Sin limitarse al uso de herramientas cartográficas para la visualización y la contextualización de investigaciones históricas, el SIG-H propone metodologías para espacializar y analizar datos de diversa naturaleza - textuales, cuantitativos, orales, etc.- en el marco de investigaciones históricas (Knowles, 2002). En este caso, por medio de un ejercicio de georreferenciación de la información espacial contenida en documentos notariales y mapas de época, se reconstruyeron dinámicas espaciales de la tenencia de la tierra en la cuenca baja del río Amaime en la segunda mitad siglo XIX. Esta articulación entre investigación archivística y SIG-H permite visualizar las vibrantes dinámicas de fragmentación experimentadas por las haciendas señoriales en el área de estudio.

Con el propósito de analizar las diferencias y los encuentros entre los diversos grupos sociales que confluyeron en este contexto agrario, se estableció una distinción espacial entre tres actores: hacendados tradicionales, hacendados empresarios y pequeños propietarios campesinos. El planteamiento de tal distinción fue resultado del análisis comparativo de diferentes unidades agrícolas en relación con las trayectorias e intereses productivos de sus propietarios. Para el objetivo acá propuesto, el pre- sente artículo se enfoca en las historias de hacendados tradicionales y pequeños propietarios. Como producto de la espacialización de datos notariales, se evidenciaron tres sectores en la cuenca baja de Amaime, cada uno de los cuales sirvió de escenario para dinámicas inmobiliarias, productivas y de poblamiento representativas de procesos más amplios experimentados por cada uno de los grupos sociales previamente distinguidos. Ubicado entre las cordilleras andinas Central y Occidental, al suroccidente de Colombia, el valle geográfico del río Cauca comprende aproximadamente 440.000 hectáreas de planicies de suelos aluviales y volcánicos a una altura promedio de $800 \mathrm{~m} \mathrm{s.} \mathrm{n.} \mathrm{m.}{ }^{5}$. El río Cauca fluye adyacente a las faldas de la cordillera Occidental que lo separa del litoral pacífico y deja un área considerable de zona plana en su margen oriental. Entre sus tributarios más importantes destaca el río Amaime, que separa las jurisdicciones municipales de El Cerrito, al norte, y Palmira, al sur (Figura 1).

\section{Tradición e innovación ganadera en la hacienda La Concepción de Amaime}

Cuando de referirse a su patrimonio rural se trata, un dicho ha acompañado a la familia Molina Cabal durante generaciones: "el que vende tierra, come tierra”. Esta expresión manifiesta la posición histórica de un grupo social particular en el contexto de las dinámicas de tenencia de la tierra en el valle

5 El departamento del Valle del Cauca fue creado en 1910. por lo cual, para evitar anacronismos, acá nos referimos al valle geográfico del río Cauca como unidad paisajística limitada a la zona plana que actualmente se circunscribe dentro de las jurisdicciones, de sur a norte, de los departamentos del Cauca, Valle del Cauca y Risaralda. 

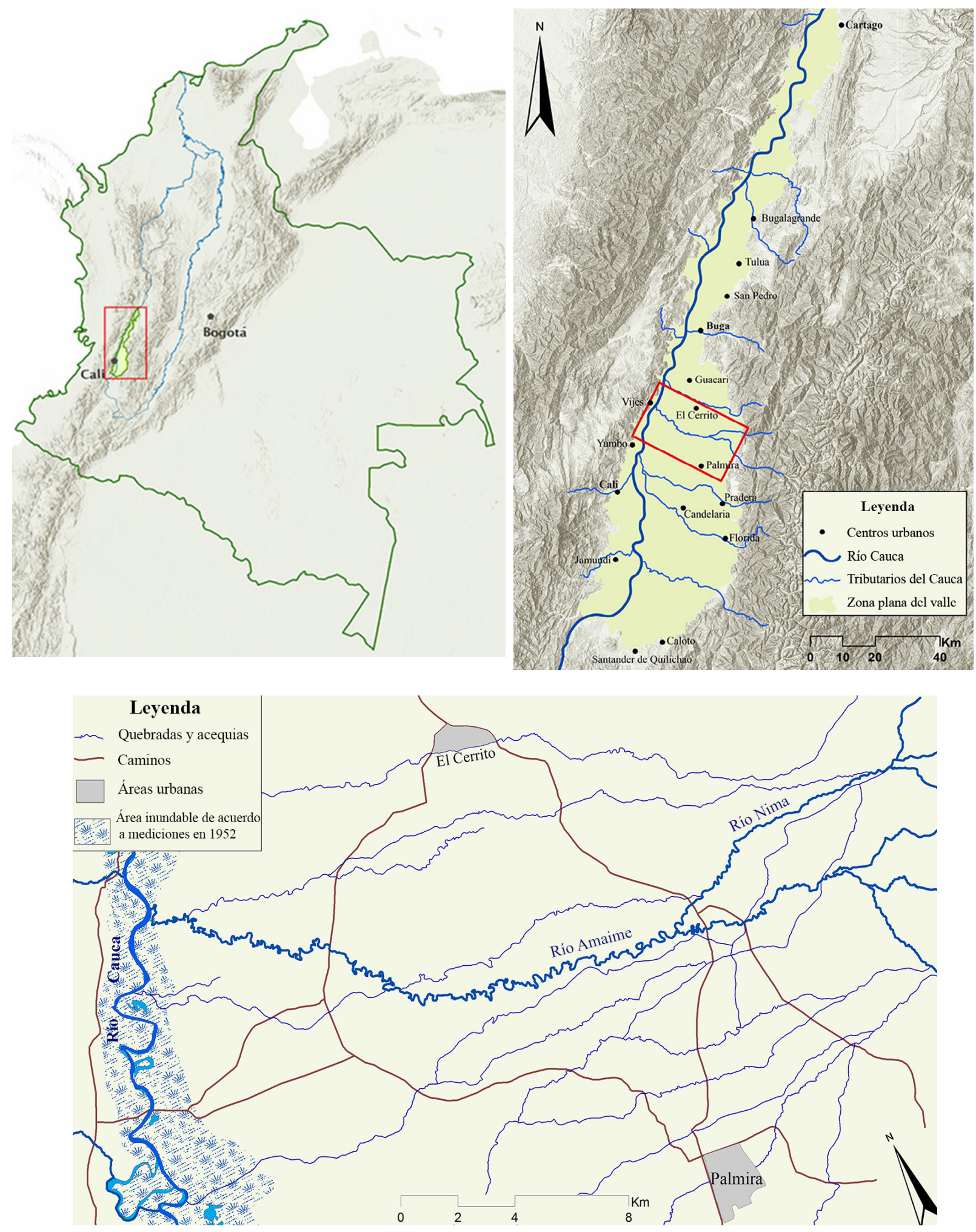

FIGURA 1.

Localización de la cuenca del río Amaime y mapa base de la ventana de estudio. Fuente: Elaboración propia. 
geográfico del río Cauca. En respuesta a las condiciones sociales, políticas y económicas instauradas desde el periodo liberal de mediados del siglo XIX, muchas familias terratenientes del Valle se vieron obligadas a replantear su relación con la tierra. Si su intención era salvaguardar su patrimonio rural amenazado por las presiones del agitado mercado inmobiliario, estas familias tenían que habitar y administrar in situ las haciendas heredadas desde tiempos coloniales. Al modificar su forma de relacionarse con la tierra y la naturaleza de esta parte del Valle, muchas familias transformaron la posición social de terratenientes ausentistas, propia de sus antepasados, por la de ganaderos y agricultores. No obstante, esta no fue la decisión de todos los miembros de familias latifundistas. Fueron numerosos los herederos y herederas que optaron por vender sus porciones de tierra para financiar negocios y su subsistencia en las ciudades. La fragmentación de haciendas abrió las puertas para que nuevos y viejos actores en la región accedieran a segmentos de estas fértiles tierras.

Las tierras al norte del río Amaime fueron nicho para algunas de las haciendas señoriales más poderosas de la región. Una de ellas fue la hacienda El Alisal, propiedad de la influyente familia Cabal Barona, de Buga. Hacia el siglo XVII, El Alisal llegó ocupar un área significativa de la zona plana en la margen oriental del río Cauca, entre las actuales jurisdicciones de Palmira y Buga, principalmente dedicada al pastoreo alrededor de lujosas casas de hacienda, capillas y trapiches con sus respectivos parches de caña de azúcar (Colmenares, 1975, p. 149). Con el tiempo, en la medida en que la familia Cabal se ramificaba por herencias y matrimonios, desde el siglo XVIII la hacienda El Alisal fue subdividida sistemáticamente en numerosas haciendas de mediano tamaño. Solo en la cuenca de Amaime, las fincas que aparecen entrado el siglo XIX en terrenos de la antigua El Alisal son, de oriente a occidente: El Paraíso, Pie de Chinche, La Providencia, La Concepción de Amaime, una versión disminuida de El Alisal y El Trejo. Debido a su ubicación exclusiva en zona plana, su lejanía de zonas inundables y un amplio acceso a las aguas del Amaime, las haciendas La Concepción y El Alisal destacan como las propiedades más privilegiadas de este grupo. Las áreas que se muestran en la Figura 2 se estimaron a partir de variadas escrituras de testamentos, permutas y compraventas en las notarías de Cali y Buga y en el archivo privado de la familia Molina.

Para mediados de siglo, La Concepción de Amaime pertenecía a José María Molina y Bárbara Cabal. En la década de 1840, la pareja amplió el tamaño de la hacienda, primero comprando un terreno de cacaotal en 1845, y después adquiriendo el lote denominado El Guaval, al otro lado del río Amaime, en 1846 (Compraventa de tierras entre Dolores Cabal y José María Molina, 1845, Archivo Privado de la Familia Molina [APFM], Cali; Compraventa de tierras entre Braulio González y José María Molina, 1846, APFM, Cali). Bárbara Cabal murió en Buga el mismo año de esta última adquisición y, en tanto titular de la heredad, su testamento revela la particular riqueza y uso agrícola de la hacienda (Tabla 1). Divididos en cuatro lotes, los derechos de tierras de La Concepción representaban el 28\% del valor total de la hacienda, seguidos del $24 \%$, que significaban 1.036 cabezas de ganado, incluyendo bueyes, caballos y mulas, y el 14\%, por 30 esclavos, cuya liberación era inminente a tan solo cinco años de la abolición en el país. En menor escala, los cultivos equivalían al 14\%, donde se destacan 8.000 árboles de cacao en buenas condiciones, por encima de escasas 6 "suertes" de caña de azúcar, 


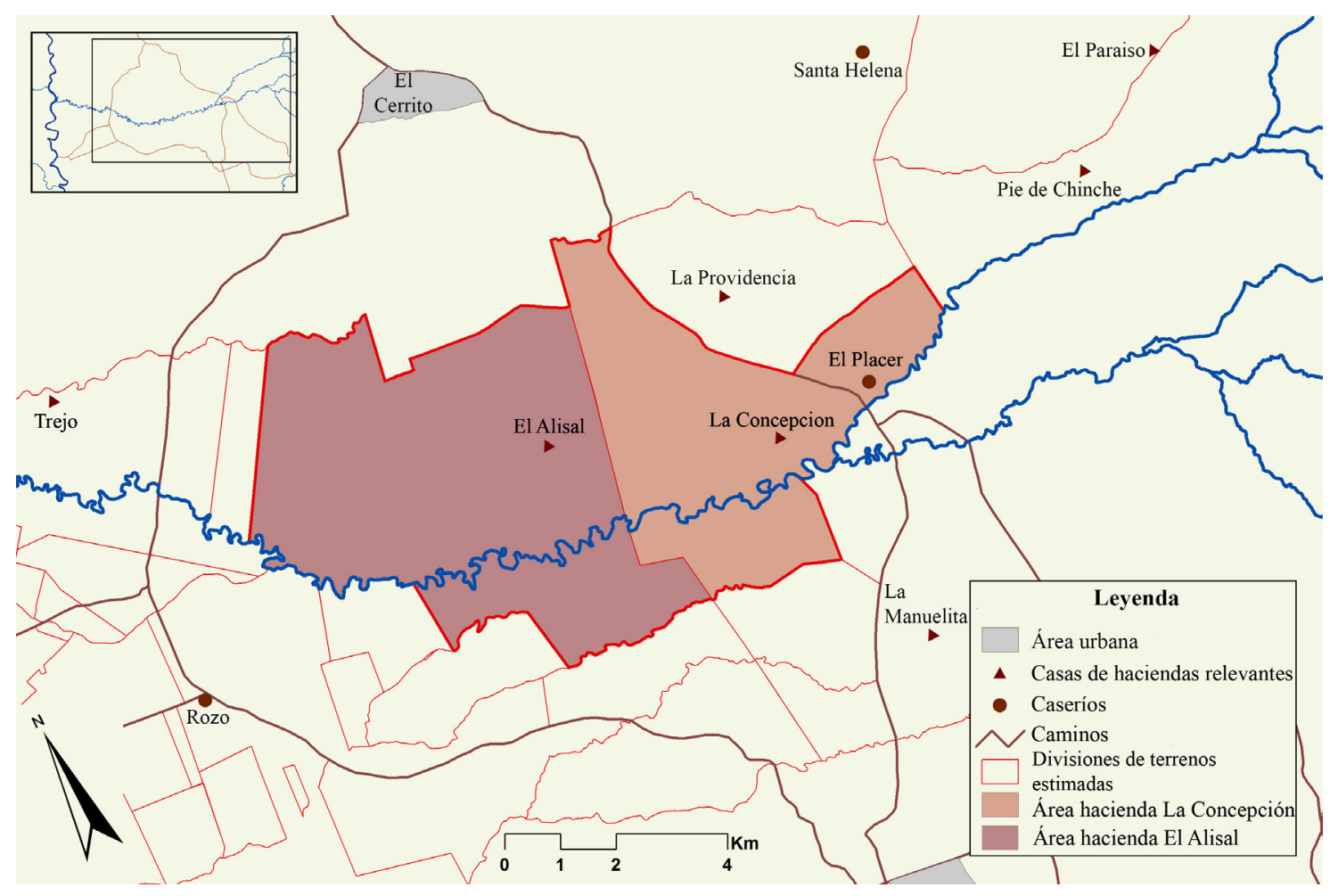

FIGURA 2.

Ubicación y linderos de las haciendas El Alisal y La Concepción de Amaime, segunda mitad del siglo XIX.

Fuente: Elaboración propia.

algunas matas de plátano y árboles frutales (Testamento de Bárbara Cabal, 1845, APFM, Cali). Pese a la presencia de un trapiche de considerables proporciones y mano de obra esclava significativa, La Concepción de 1845 no privilegió el cultivo de caña de azúcar.

Tras su muerte en 1863, las propiedades de José María Molina fueron distribuidas en partes iguales entre sus cuatro descendientes: María Josefina, Bernardino, Enrique y Adelaida Molina Cabal. Su testamento evidencia importantes mejoras a la hacienda con respecto a la situación en 1845 (Tabla 1). Durante estos años, José María invirtió significativamente en los cultivos de caña de azúcar, cuyo avalúo pasó de 180 pesos en 1845 a
900 pesos en 1863, mientras que la plantación de cacao disminuyó en un $42 \%$ su precio. Este viraje productivo se vio respaldado por importantes inversiones de infraestructura en el trapiche, que pasó de avaluarse de 500 a 1.400 pesos, a las que se suman nuevas herramientas y espacios para procesar el cacao. Sin embargo, el sector de la hacienda que más atención recibió por parte de Molina fue el ganadero. Además del aumento en el precio de las cabezas de ganado de 9.084 pesos en 1845 a 14.115 pesos en 1863, Molina, al igual que muchos otros terratenientes de la zona, invirtió en pastos africanos, particularmente en pastos Guinea (Megathyrus maximus) (Testamento de José María Molina, 1863, AHB, Buga). Los pas- 
tos Guinea, junto con los pastos Pará (Brachiara incentivar la eficiencia del engorde de ganado, mutica), tuvieron gran acogida entre los ganade- aunque también para consolidar la apertura de ros colombianos durante la segunda mitad del si- potreros y aumentar el precio de las tierras (Van glo XIX, convirtiéndose en insumo crucial para Ausdal, 2009b).

\section{TABLA 1. AVALÚO DE LOS BIENES DE LA HACIENDA LA CONCEPCIÓN DE AMAIME EN 1845 Y 1863}

\begin{tabular}{|c|c|c|c|c|c|c|}
\hline \multirow[b]{2}{*}{ Ítem } & \multicolumn{3}{|c|}{1845} & \multicolumn{3}{|c|}{1863} \\
\hline & $\begin{array}{c}\text { Precio } \\
\text { desagregado } \\
\text { (pesos) }\end{array}$ & $\begin{array}{l}\text { Precio } \\
\text { agregado } \\
\text { (pesos) }\end{array}$ & Porcentaje & $\begin{array}{c}\text { Precio } \\
\text { desagregado } \\
\text { (pesos) }\end{array}$ & $\begin{array}{c}\text { Precio } \\
\text { agregado } \\
\text { (pesos) }\end{array}$ & Porcentaje \\
\hline Derechos de tierra & 10.636 & 10.636 & $28 \%$ & 8.484 & 8.484 & $28 \%$ \\
\hline Esclavos & 5.461 & 5.461 & $14 \%$ & & & \\
\hline Edificios vivienda & 3.850 & \multirow{5}{*}{4.764} & \multirow{5}{*}{$12 \%$} & 2.440 & \multirow{5}{*}{4.510} & \multirow{5}{*}{$15 \%$} \\
\hline Trapiche & 500 & & & 1.400 & & \\
\hline Otra infraestructura & 271 & & & 280 & & \\
\hline Cercas & & & & 120 & & \\
\hline Herramientas & 143 & & & 265 & & \\
\hline Ganado (agregado) & 9.084 & \multirow{2}{*}{9.084} & \multirow{2}{*}{$24 \%$} & 12.675 & \multirow{2}{*}{14.115} & \multirow{2}{*}{$47 \%$} \\
\hline Pastos importados & & & & 1.440 & & \\
\hline Caña de azúcar & 180 & \multirow{5}{*}{5.312} & \multirow{5}{*}{$14 \%$} & 900 & \multirow{5}{*}{3.020} & \multirow{5}{*}{$10 \%$} \\
\hline Árboles de cacao & 5.000 & & & 2.100 & & \\
\hline Plátano & 82 & & & & & \\
\hline Huerta & 50 & & & & & \\
\hline Árboles frutales & & & & 20 & & \\
\hline Otros & 2.950 & 2.950 & $7 \%$ & & & \\
\hline Totales & 38.207 & & $100 \%$ & 30.124 & & $100 \%$ \\
\hline
\end{tabular}

Fuente: elaboración propia con base en el Testamento de Bárbara Cabal, junio 17 de 1845, APFM, y el Testamento de José María Molina, agosto $1^{\circ}$ de 1863, Archivo Histórico de Buga (AHB). 
Durante los 18 años posteriores a la muerte de su esposa, José María Molina enfocó su atención en administrar y mejorar el funcionamiento de la hacienda, para lo cual debió sobrellevar la transición de la forma de trabajo esclavista a la asalariada ${ }^{6}$. La Tabla 1 detalla la dinámica de cambio en el avalúo de la hacienda La Concepción entre estos dos momentos, aunque se evidencia que para 1863 Molina había vendido una parte de las tierras de las cuales no contamos con registro alguno. En el intervalo, Molina y sus hijos pasaron a habitar la hacienda, desde donde pudieron administrar sus actividades agropecuarias. En paralelo, los antiguos esclavos de la hacienda probablemente pasaron a poblar el caserío de El Placer, ubicado en el encuentro de caminos que, cruzando el río Amaime, comunican a Palmira con El Cerrito (Figura 3). La forma de vida de propietarios ausentistas en la familia Molina Cabal había quedado en el pasado, así como las relaciones laborales esclavistas en que se basaba este sistema de tenencia de la tierra.

Siguiendo el legado de su padre, los hermanos Enrique y Bernardino Molina Cabal se radicaron en la casa de campo heredada en 1863 y continuaron las actividades agropecuarias, con principal ahínco en la ganadería. En contraste, sus hermanas María Josefina y Adelaida eligieron mantener sus vidas en la ciudad, para lo cual negociaron rápidamente sus partes de la hacienda. El mismo año de 1863, María Josefina y su esposo Rafael Rebolledo vendieron a Enrique y Bernardino su fracción de la

6 Aunque desafortunadamente no contamos con fuentes para analizar en detalle esta dimensión, se puede decir que esta transición laboral estuvo asociada al trabajo agrícola dentro de las haciendas en forma de peonaje (Mejía Parado, 1993). hacienda por 10.000 pesos, pues, en su juicio, "no les es, en manera alguna útil y conveniente administrar los proindivisos" (Compraventa de tierras entre María Josefina, Bernandino y Enrique Molina, 1863, APFM, Cali). En paralelo, los hermanos también le hicieron una oferta conjunta a Adelai$\mathrm{da}$, a quien otorgaron los derechos de la casa de su difunto padre en Buga más 1.200 pesos, pues "los otorgantes tienen otras fincas y les conviene administrar proindiviso esa parte de herencia" (Compraventa de tierras entre Adelaida, Bernandino y Enrique Molina, 1863, APFM, Cali). Como en numerosos casos rastreables en las notarías, las hijas de familias terratenientes solían ser las primeras en vender sus derechos de tierras heredados, en muchos casos para sostener vidas urbanas.

A lo largo de las siguientes décadas, los hermanos Molina Cabal continuaron administrando la hacienda sin indicio alguno de arriendos o subdivisiones. Como primogénito, a Bernardino le correspondió la casa de la hacienda, el trapiche y los terrenos circundantes, mientras que Enrique quedó a cargo de la sección norte de la propiedad, llamada El Albión (Figura 3). No obstante, en la práctica parece que los dos hermanos manejaron conjuntamente la operación de La Concepción, de acuerdo con indicios en la libreta de cuentas cotidianas de Enrique Molina (Libreta de Enrique Molina, 1876-1887, APFM, Cali). Hacia el costado oriental, la sección llamada El Placer continuó bajo título de la familia Molina, como se puede entrever en menciones esporádicas dentro de la documentación, sin embargo, en cierto momento estas tierras pasarían a ser tituladas a pequeños propietarios asociados a las dinámicas comerciales en este cruce de caminos. 


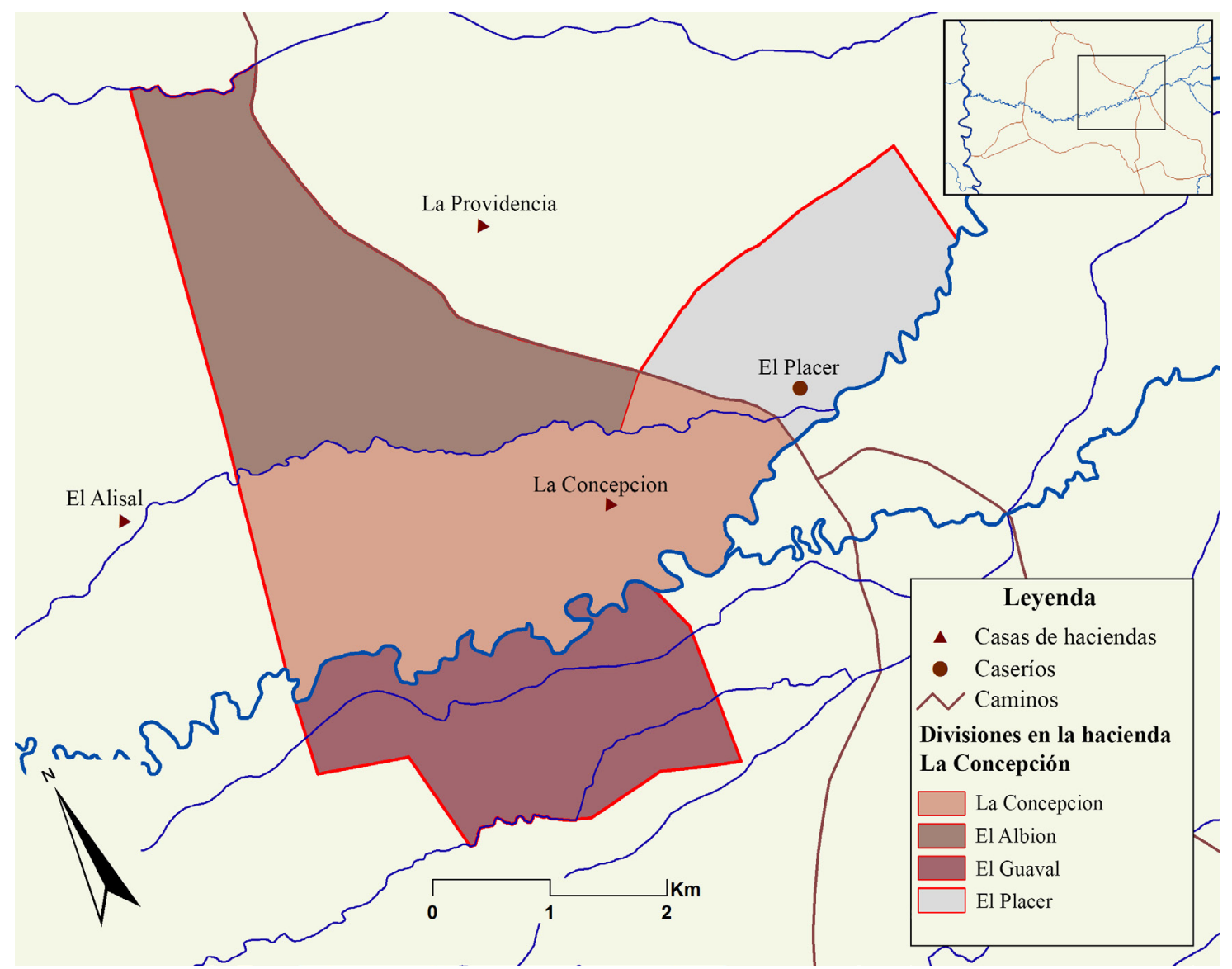

FIGURA 3.

Distribución de las tierras dentro de la hacienda La Concepción de Amaime, 1890. Fuente: Elaboración propia.

Los documentos del archivo privado de la familia Molina permiten evidenciar el eminente uso ganadero que se le dio a las tierras de la hacienda La Concepción. Las libretas personales de Enrique Molina hablan de un constante movimiento de animales, principalmente caballos y mulas para venta y alquiler. La demanda de animales de tracción y de carga en el Valle era constante, bien fuera como fuentes de energía para mover los trapiches artesanales o como medios para transportar mercancías y alimentos dentro y fuera de la región. Dadas las condiciones geográficas a que se enfrentaban estos animales, los comerciantes exigían mulas y caballos de carga de alta calidad, capaces de franquear pantanos y zanjones cenagosos, e incluso de cruzar las accidentadas cordilleras hacia el océano o hacia el interior. Las mulas de Molina variaban sus precios entre 16 y 40 pesos cada una, dependiendo de su uso. En promedio, entre 1878 y 1886 sus cuentas registran al rededor de 280 caballos y 120 mulas por año (Libreta de Enrique Molina, 18761887, APFM, Cali).

Bernardino Molina solo tuvo una hija, por eso cuando cayó gravemente enfermo, en 1892, él y su hermano Enrique decidieron hacer un contrato para redistribuir sus títulos y proteger a futuro la integridad de la hacienda La Concepción (Per- 
muta entre Bernardino y Enrique Molina, 1892, APFM, Cali). Así, El Albión quedó a nombre Bernardino, mientras que la parte sur de la hacienda, de mayor valor y extensión, pasó a manos de Enrique y, eventualmente, de sus tres hijos y sus dos hijas. Por medio de esta transacción, los hermanos Molina buscaron proteger los terrenos de su hacienda patrimonial para la siguiente generación, dadas las condiciones del mercado de tierras en situaciones de sucesión, cuando las tierras pasaban a ser un bien comercializable acorde a los intereses individuales de los hermanos. Incluso tras la muerte de su hermano mayor en 1893, Enrique continuó con su mirada puesta en los negocios ganaderos de El Albión donde, de acuerdo con un inventario elaborado por sus propias manos en 1894, se contabilizan 1.034 cabezas de ganado pastando en 548 hectáreas, buena parte de ellas sembradas con pastos Guinea y Pará, además de 20 suertes de caña (Cuentas de la hacienda El Albión, 1894, APFM, Cali).

En 1897 murió Enrique Molina y La Concepción pasó a manos de sus hijos, quienes, perpetuando la tradición familiar, mantuvieron la producción ganadera de la hacienda, mientras sus hijas se asentaron en la ciudad de Cali. Esta nueva generación se encargaría de introducir ganado lechero a la hacienda, actividad emergente en la región desde principios del siglo XX gracias a la introducción de razas cebú y Holstein en la hacienda La Manuelita, colindante de La Concepción, de acuerdo con la narrativa de Phanor Eder sobre el papel pionero de su padre, Santiago, fundador del ingenio Manuelita (Eder, 1981, p. 467). Este viraje productivo hacia la actividad lechera mostró ser rentable en décadas siguientes. Prueba de ello fue la decisión de los descendientes Molina de transformar el antiguo trapiche de la hacienda en una instalación de ordeño en 1922 (Juicio de sucesión de los bienes de Enrique Molina, 1922, APFM, Cali). Con la consolidación del Valle del Cauca como enclave agroindustrial a partir de la década de 1950, la presión sobre las tierras de La Concepción cambió de contexto y de características, particularmente desde la fundación del ingenio Providencia, colindante por su costado noroccidental. Los actuales descendientes de la familia Molina aún repiten "el que vende tierra, come tierra" cuando les preguntan por la permanencia de su patrimonio familiar a orillas del río Amaime.

\section{Fragmentación y poblamiento campesino en el área de La}

\section{Torre}

Desde La Concepción, cruzando el río Amaime y siguiendo corriente abajo hacia su desembocadura en el río Cauca, se encuentran las tierras conocidas como La Torre, actual corregimiento de Rozo. En la medida en que los humedales al rededor del Cauca se estrechaban hacia las desembocaduras de Amaime y El Cerrito, las opciones para cruzar a la banda occidental se facilitaban considerablemente, haciendo de esta una zona ideal para el comercio regional. El paso de La Torre fue uno de los puntos históricos de conexión entre las dos bandas del río Cauca (Banderas, 1944), y también como área de cruce de caminos entre Palmira, El Cerrito y Buga. En el siglo XIX, tales condiciones geográficas atrajeron la atención de múltiples actores vinculados al comercio, pero también de agricultores sin mayores opciones de adquirir tierras en el apretado mosaico de haciendas del Valle. La Torre fue escenario predilecto del agitado mercado inmobiliario en la 
jurisdicción de Palmira a causa del contraste entre crecimiento demográfico y desigualdad en el acceso a la tierra; la trayectoria de las haciendas allí ubicadas representa un panorama muy diferente al previamente explorado al norte del río Amaime.

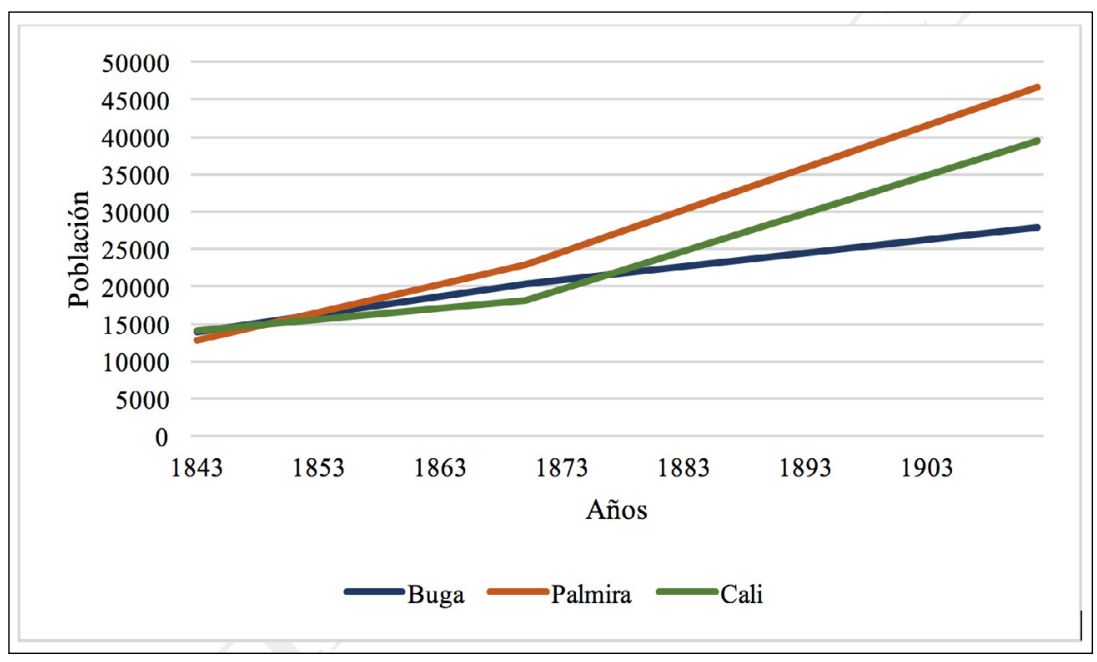

FIGURA 4.

Cantidad de población agregada por cantones entre 1843 y 1912.

Fuente: Elaboración propia con base en datos de Gobierno Nacional de Colombia (1912, p. 49); Mejía Parado (1993, pp. 120-121 y 125-126); y Valdivia Rojas (1980, pp. 108-109).
La ola de colonización interna en el valle geográfico del Cauca durante el siglo XIX siguió dinámicas particulares con respecto a los procesos contemporáneos en la región antioqueña. Si bien hubo presencia de migración de campesinos blancos y mestizos de otras regiones hacia el Valle, principalmente en la parte norte, el proceso de colonización en la zona plana estuvo protagonizado por la población afrocolombiana previamente asociada al mercado esclavista (Almario García, 2013; Valencia Llano, 2016). Este proceso de poblamiento había empezado desde el siglo XVIII, pero la abolición de la esclavitud en 1851 provocó su aceleración, tal como lo muestran las cifras demográficas proporcionadas por Luis Valdivia y Oscar Almario. De 92.901 habitantes en el Valle para 1843, se pasó a 169.385 en 1870, que se estima llegaron a ser, en 1890, al rededor de 200.000; Cali y Palmira fueron los epicentros de mayor densidad poblacional (Figura 4) (Almario García, 2013; Valdivia Rojas, 1980).
Como efecto de este crecimiento demográfico, los asentamientos en la zona plana del Valle adquirieron sistemáticamente el estatus de cantones, pueblos, municipios o corregimientos. En el área entre Buga y Santander de Quilichao emergieron los pueblos de Palmira en 1824, Candelaria, El Cerrito y Florida en 1864, Pradera en 1870 y Miranda en 1899 (Banderas, 1944). Aunque con algunos precedentes en querellas de tiempos coloniales, la formación de estos municipios tuvo lugar en el marco del federalismo liberal y la emergencia de procesos de representación local bajo el control de autoridades civiles y terratenientes (Mejía Parado, 1993, p. 118). En los sectores periféricos al sur de Palmira, incluso se evidencia la donación de tierras por parte de terratenientes para la concentración urbana de la población campesina, evitando así su libre movimiento por tierras de las haciendas y facilitando su vinculación laboral en forma de peonaje (Almario García, 2013, p. 67). El acentuado crecimiento en el cantón de Palmira (Figu- 
ra 4) estuvo vinculado al floreciente mercado del tabaco, cultivado por pequeños productores, por lo general, arrendatarios en tierras de haciendas (Valdivia Rojas, 1992, p. 42), y comercializado nacional e internacionalmente por empresarios locales y extranjeros (Giraldo Hoyos, 2018, p. 107). Descrita por el catedrático vallecaucano Luciano Rivera y Garrido (1886), Palmira "es la menos antigua de las poblaciones principales del Estado del Cauca, $\mathrm{y}$ al propio tiempo una de las que han progresado más rápidamente, debido al espíritu industrioso de sus hijos, que en oportunidad supieron aprovechar la feracidad del suelo, adecuado, sobre todo, para el cultivo del tabaco" (p. 55).

Como consecuencia de la explosión demográfica en Palmira, el sector de La Torre experimentó una diversificación de pequeños propietarios a expen- sas de la fragmentación de una hacienda. Antes de la fundación de Palmira, este sector hacía parte de la poderosa hacienda Llanogrande, asociada a la familia Cobo desde el siglo XVII, después fue adquirida por la Compañía de Jesús, a la que le fue expropiada en el siglo XVIII, para pasar a ser rematada entre terratenientes de la región. Para la segunda mitad del XIX, todavía encontramos a una descendiente de esta familia como propietaria de una parte de este antiguo latifundio: Carmen Cobo y su esposo José Vásquez Córdoba poseían los derechos de tierras de la hacienda La Torre. La poca información que tenemos sobre esta familia y su hacienda viene de cuantiosas transacciones de tierras efectuadas en las notarías de Cali, ciudad de residencia de la familia Vásquez Cobo, desde la década de 1870, las cuales están georreferenciadas en la Figura 5.

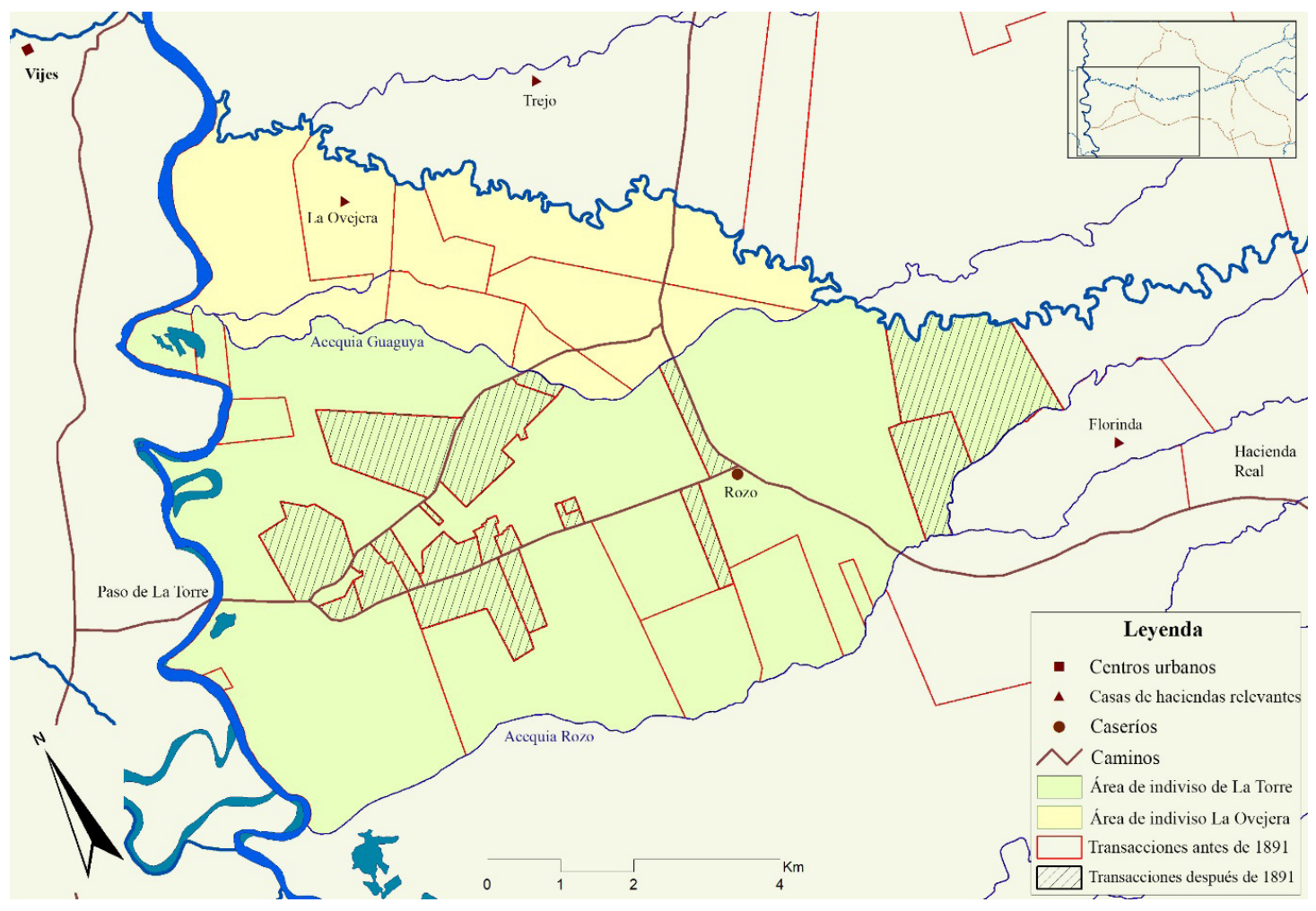

FIGURA 5.

Distribución estimada de tierras en el sector de La Torre, con distinción de transacciones antes y después de 1891.

Fuente: Elaboración propia. 
En diciembre de 1891 muere en Cali José Vásquez Córdoba, pocos años después de su esposa Carmen, y es entonces cuando sus herederos deciden fragmentar y vender por lotes la antigua hacienda La Torre, la cual pasa a ser considerada como terreno proindiviso. Producto del escenario de tenencia de la tierra en el Valle, la figura de proindivisos o indivisos fue común en la región, entendidos estos como remanentes de antiguas haciendas que, tras procesos de loteo, ofrecieron oportunidades de acceso a la tierra a nuevos propietarios con variados poderes adquisitivos (Gutiérrez R., 1981; Ramírez, 1984). Desde décadas anteriores, Vásquez Córdoba ya había vendido una porción importante de la parte sur de La Torre a Joaquín de Caicedo y Caicedo, quien, a su vez, vendió primero 576 hectáreas por 9.000 pesos a José María Tejada (Contrato de compraventa entre Caicedo y Tejada, 1880, Archivo Histórico de Cali [AHC], Notaría Primera, Cali), y en 1880, otro lote al general Tomás Rengifo por 16.000 (Contrato de compraventa entre Caicedo y Rengifo, 1875, AHC, Notaría Primera, Cali) (Figura 5). La presencia de miembros influyentes en La Torre, entre los cuales sobresale el general liberal y exgobernador de la Provincia de Antioquia, Tomás Rengifo, nos permite hablar de la diversidad de actores que confluyeron en esta locación, pues después de 1891 otros serían los compradores predilectos en este proindiviso.

La Figura 5 representa la distribución aproximada de tierras en La Torre para finales del siglo XIX con base en datos notariales extraídos principalmente de las notarías primera y segunda de Cali. En la esquina nororiental, entre la acequia Guaguya y el río Amaime, se ubicaba la antigua hacienda La Ovejera (en amarillo en el mapa), la cual, de acuerdo con un mapa de agrimensura elaborado en 1883, fue dividida en siete secciones (Plano de la
Antigua hacienda La Ovejera, 1883, AHC, Notaría Segunda, Cali). Al sur, los terrenos de La Torre (en verde en el mapa) se extendían de norte a sur entre la acequia Guaguya y el zanjón de Rozo, y de oriente a occidente entre el río Cauca y el proindiviso de Hacienda Real. Los polígonos achurados simbolizan aquellos lotes vendidos por los descendientes de la familia Vásquez Cobo después de 1891, año de muerte del patriarca. La mayoría de estas propiedades, mayoritariamente colindantes con caminos, escasamente superaban las $120 \mathrm{hec}$ táreas, con un promedio de valor de 47 pesos por hectárea. Entre los compradores de estos lotes no sobresalen apellidos prestigiosos y muchos de ellos se declaraban residentes de diferentes pueblos (en los casos en que se daba esta información): diez eran vecinos de Palmira, cinco de Cali, cuatro de Yumbo y dos de Vijes.

Información parcial sobre usos del suelo en los documentos notariales evidencian el predominio de pastos para ganado en La Torre para finales del siglo XIX, aunque también hay menciones de cultivos de cacao, plátano y maíz. La presencia de pequeños cultivos de subsistencia y comerciales tiene sentido dentro de la limitada escala de los lotes identificados, revelando así una dinámica agropecuaria asociada al caserío de Rozo y al crecimiento poblacional de actores campesinos en la zona. Si bien la presencia de un pequeño núcleo poblacional de comunidades afrodescendientes en el punto de Rozo se remonta a épocas anteriores (Crist, 1952, p. 28; Arévalo, Ruan \& Sandoval, 1959), la evidencia notarial nos permite pensar que es hasta la desintegración de la antigua hacienda La Torre que estos actores tuvieron acceso legal a las tierras al rededor de los caminos. Este proceso agrícola y de poblamiento continuaría durante el siglo XX hasta la formación del corregimiento de Rozo den- 
tro del municipio de Palmira, el cual hoy alberga un parche de policultivo dentro del dominio del monocultivo de caña de azúcar.

Basados en el autosustento y la producción de pequeños cultivos comerciales, los sistemas agrícolas del campesinado vallecaucano cumplieron con un rol fundamental en el proceso de consolidación regional, como fuente de alimentos para los pujantes centros urbanos y como proveedores de materias primas para mercados comerciales de productos agrícolas tales como tabaco, caña, añil y cacao. Tomemos por ejemplo el lote adquirido por Rafael Antonio Sánchez en 1896, ubicado sobre el camino de Rozo al paso de La Torre (Figura 5), por un valor de 3.000 pesos. Cercano al tamaño promedio de terrenos en el proindiviso de La Torre, el contenido de este inmueble puede darnos una idea de las condiciones materiales de las unidades agrícolas en este sector. En un área estimada de 30 hectáreas, el lote incluía cuatro plazas de caña, algunos árboles de cacao, dos plazas de plátano, una plaza de maíz, seis plazas de pastos Guinea y Pará, dos cerdos, un guadual, un trapiche de piedra y herramientas (Contrato de compraventa entre Orejuela y Sánchez, 1896, AHC, Notaría Primera, Cali). El buen estado del terreno y sus construcciones explican el alto precio pagado por Sánchez, al tiempo que evidencian las condiciones materiales de una finca con una producción activa, diversificada y estratégicamente localizada con relación al circuito comercial de Rozo. Se puede suponer que este no era un caso aislado y que, en diferentes escalas, los terrenos en La Torre lograron transformar terrenos previamente dominados por el pastoreo para el cultivo diversificado de alimentos.

\section{Conclusiones}

El análisis detallado de las haciendas vallecaucanas desde una perspectiva espacial e histórica nos permite dimensionar la trayectoria del sistema de tenencia de la tierra dentro de un contexto de constante tensión entre tradición agraria y cambio social. Con dinámicas opuestas, las dos áreas abordadas en la cuenca del río Amaime representan procesos históricos más amplios que tuvieron lugar en el valle geográfico del río Cauca durante la segunda mitad del siglo XIX. Las haciendas de esta región siguieron caminos desiguales dependiendo de sus ubicaciones geográficas y de las decisiones individuales en momentos de sucesión patrimonial. Ya fuese vendiendo o conservando, los miembros de familias propietarias tradicionales fueron actores decisivos en la configuración del paisaje agropecuario del Valle del Cauca.

El caso de La Concepción de Amaime ilustra los pasos seguidos por una familia que, pese a su legado señorial, desarrolló un vínculo cercano con la tierra que le permitió mantener relativamente íntegros los linderos de su hacienda y sostener una fuente de soporte económico por generaciones. Este vínculo se basó en la decisión de los herederos hombres de habitar y administrar la hacienda, aplicando esporádicas mejoras tanto de infraestructura como agropecuarias. El espacio restringido para las mujeres en el contexto rural hizo que las herederas eligieran en general la vida urbana. Sin tomar mayores riesgos en nuevas actividades productivas, los herederos Molina optaron por conservar el eminente uso ganadero de La Concepción, el cual buscaron incentivar inicialmente por medio de inversiones en pastos Guinea y Pará, y luego con la introducción de especies y la remodelación 
de infraestructuras para producción lechera. Aunque carecemos de datos de producción general, es justo pensar que gran parte de las ganancias estuvieron destinadas al sostenimiento de La Concepción y de la familia Molina. Tal desempeño contrasta con el auge de un pensamiento empresarial y sus ambiciones de expansión en la región, incluso en propiedades vecinas a la hacienda, como las tierras de la hacienda Manuelita donde el empresario ruso-estadounidense Santiago Eder llevó a cabo diferentes iniciativas productivas con propósitos de exportación desde 1864 (Giraldo Hoyos, 2018).

A escasos ocho kilómetros de La Concepción, la propiedad de la familia Vásquez Cobo vivió una historia muy diferente. En el caso de la hacienda La Torre, su ubicación geográfica condicionó en gran medida el proceso de fragmentación territorial que venía de tiempo atrás. En un espacio de palpitante tránsito tanto entre norte y sur de la banda oriental, como entre occidente y oriente del río Cauca, la hacienda de La Torre, como otras vecinas, vivió la presión de la colonización interna. Acorde a la evidencia presentada, la familia Vásquez no replanteó su relación con la tierra, la cual, por el contrario, fue comercializada para el beneficio de otro tipo de negocios. Gracias a esta decisión, en buena parte motivada por la demanda de lotes, actores marginados por el orden territorial establecido pudieron acceder a tierras fértiles y estratégicamente ubicadas para el ejercicio de actividades agropecuarias. Segregados a franjas de caminos, humedales y piedemontes, las comunidades campesinas - afrodescendientes y mestizasse abrieron campo en el paisaje agropecuario del valle geográfico, del cual fueron tanto actores activos como trabajadores asalariados y agricultores en pequeñas propiedades. La ventana temporal acá analizada corresponde a una pequeña parte del lar- go proceso de fragmentación vivido en el actual corregimiento de Rozo, el cual amerita mayor profundización como caso de resistencia agrícola en el contexto del monocultivo azucarero.

Estos cambios y permanencias en el sistema de tenencia de la tierra no afectaron en mayor medida las dinámicas de uso del suelo en un primer momento, pues tanto en La Concepción como en La Torre siguió predominando la actividad ganadera, con algunas excepciones, según las fuentes consultadas. Sin embargo, a partir de principios del siglo XX los sectores de Rozo y de La Torre sobresaldrían por su uso de prácticas agrícolas diversificadas (Arévalo et al., 1959), mientras que, para finales del siglo XX, las tierras de La Concepción reducirían paulatinamente sus zonas de pastoreo para el cultivo de caña de azúcar ante la presión ejercida por el ingenio Providencia. Paradójicamente, en el siglo XX tanto las familias hacendadas como los pequeños agricultores enfrentaron las amenazas de la agroindustria sobre sistemas agrarios tradicionales. La expansión del monocultivo de caña reemplazó sistemáticamente la ganadería como uso dominante del suelo en el valle geográfico, al tiempo que los espacios agrícolas para comunidades campesinas, afrodescendientes e indígenas continuaron siendo marginados.

El análisis de Raymond Crist sobre la relación entre un sistema de tenencia de la tierra desigual y patrones improductivos de uso del suelo no estaba equivocado: el sistema de la hacienda señorial restringió el uso del suelo del Valle a la ganadería extensiva. Sin embargo, el modelo de hacienda que él retrató sufrió importantes cambios en el siglo XIX, con los cuales la lógica de tenencia, definida por el prestigio sobre la productividad, se vio alterada. Incluso las haciendas tradicionales tuvieron 
que replantear sus dinámicas productivas para sobrevivir a los cambios históricos desatados por el liberalismo decimonónico. La versión de Crist del sistema de la hacienda vallecaucana como "una camisa de fuerza que llevó a una aguda situación de artritis social y anemia económica" (Crist, 1952, p. 26) cobra sentido cuando entendemos que sus ideales de uso de la naturaleza estaban enfocados hacia el progreso económico, sin tener en cuenta los límites ambientales y las formas agropecuarias adaptadas a estas condiciones.

La articulación de fuentes documentales y herramientas digitales de análisis espacial brinda valiosas oportunidades para revisitar viejos paradigmas de investigación. En este caso, nos permitió analizar en pequeña escala casos particulares dentro de procesos históricos generalmente obnubilados por generalizaciones. Los archivos notariales ofrecen cuantiosa información espacial con gran potencial de ser georreferenciada, sin embargo, tal esfuerzo requiere de un manejo documental apropiado para estas fuentes, por lo general dispersas y sin catalogación. Una sistematización y georreferenciación completa de documentos notariales podría contribuir a reconstruir registros catastrales históricos en regiones de Colombia que, por circunstancias propias del conflicto armado desde mediados del siglo $\mathrm{XX}$, nunca se llevaron a cabo. 


\section{Bibliografía}

\section{Archivos consultados}

Archivo Privado de la Familia Molina (APFM).

Archivo Histórico de Cali (AHC).

Archivo Histórico de Buga (AHB).

\section{Fuentes de archivo}

Compraventa de tierras entre Adelaida, Bernandino y Enrique Molina. (1863).

[Escritura]. APFM. Cali.

Compraventa de tierras entre María Josefina, Bernandino y Enrique Molina. (1863).

[Escritura]. APFM. Cali.

Compraventa de tierras entre Braulio González y José María Molina. (1846).

[Escritura]. APFM. Cali.

Compraventa de tierras entre Dolores Cabal y José María Molina. (1845). [Escritura]. APFM. Cali.

Contrato de compraventa entre Caicedo y Rengifo. (1875). [Escritura]. AHC, Notaría Primera. Cali.

Contrato de compraventa entre Caicedo y Tejada. (1880). [Escritura]. AHC, Notaría Primera. Cali.

Contrato de compraventa entre Orejuela y Sánchez (1896). [Escritura]. AHC, Notaría Primera. Cali.

Cuentas de la hacienda El Albión. (1894). [Escritura]. APFM. Cali.

Juicio de sucesión de los bienes de Enrique Molina. (1922). [Escritura]. APFM. Cali.

Permuta entre Bernardino y Enrique Molina. (1892). [Escritura] APFM. Cali.

Plano de la Antigua hacienda La Ovejera. (1883). [Mapa]. AHC, Notaría Segunda. Cali.

Testamento de Bárbara Cabal. (1845). [Escritura]. APFM. Cali.

Testamento de José María Molina. (1863). [Manuscrito]. AHB. Buga.

\section{Fuentes impresas}

Almario García, Ó. (2013). La configuración moderna del Valle del Cauca, Colombia, 18501940. Espacio, poblamiento, poder y cultura. Popayán: Editorial Universidad del Cauca.

André, É. F. (2013). La América Equinoccial (Colombia, Ecuador, Perú). En P. Navas Sanz de Santamaría (ed.), Colombia en Le Tour du Monde (vol. 2, pp. 7-22). Bogotá: Villegas Editores. 
Arévalo, E., Ruan, F. \& Sandoval, A. (1959). Estudio socio-económico del corregimiento de Rozo, Municipio de Palmira. Acta Agronómica, 9(1-2), 51-112.

Banderas, P. A. (1944). Diccionario geográfico, industrial y agrícola del Valle del Cauca. Buenos Aires: Instituto Banderas.

Colmenares, G. (1975). Cali: terratenientes, mineros y comerciantes. Siglo XVIII. Cali: Universidad del Valle.

Crist, R. E. (1952). The Cauca Valley, Colombia: Land Tenure and Land Use. Baltimore MD: Waverly Press.

Delgadillo Vargas, O. L. (2014). La caña de azúcar en la historia ambiental del valle geográfico del río Cauca (1864-2010). (Tesis doctoral). Pontificia Universidad Javeriana, Bogotá, Colombia.

Eder, P. J. (1981). El fundador Santiago M. Eder. Bogotá: Flota Mercante Grancolombiana.

Escorcia, J. (1982). Haciendas y estructura agraria en el Valle del Cauca 1810-1850. Anuario Colombiano de Historia Social y de la Cultura, 10, 119-138.

Giraldo Hoyos, M. (2018). Owning Land, Appropriating Nature. The Configuration of an Agricultural Landscape in the Cauca River Valley, South Western Colombia, 1864-1901. (Tesis de maestría). University of Saskatchewan, Saskatoon, Canadá.

Gobierno Nacional de Colombia. (5 de marzo de 1912). Censo General de la República de Colombia. Bogotá: Imprenta Nacional.

Guillén, F. (1979). El poder político en Colombia. Bogotá: Punta de Lanza.

Gutiérrez R., G. (1981). El indiviso de Guabas, 1651-1937. (Tesis de licenciatura). Universidad del Valle, Cali, Colombia.

Hecht, S. B. (1993). The logic of livestock and deforestation in Amazonia. Bioscience, 43(10), 687-695.

Holton, I. F. (1857). New Granada: Twenty Months in the Andes. New York: Harper \& Brothers.

Knowles, A. K. (Ed.). (2002). Past Time, Past Place: GIS for history. Redlands, California: ESRI Press.

LeGrand, C. (1988). Colonización y protesta campesina en Colombia (1850-1950). Bogotá: Universidad Nacional de Colombia.

Llanos Vargas, H. (1979). Japio: modelo de hacienda colonial en el Valle del Cauca (s. XVI-XIX. Historia y Espacio, 2, 8-73. https://doi.org/10.25100/hye.v0i2.4714

Marull, J., Delgadillo, O., Cattaneo, C., La Rota, M. J. \& Krausmann, F. (2018). Socioecological transition in the Cauca river valley, Colombia (1943-2010): Towards an energy-landscape integrated analysis. Regional Environmental Change, 18(4), 1073-1087. https://doi.org/10.1007/s10113-017-1128-2.

Mejía Parado, E. (1993). Origen del campesino vallecaucano. Cali: Universidad del Cauca. 
Mina, M. (1975). Esclavitud y libertad en el valle del río Cauca. Bogotá: Publicaciones de la Rosca.

Montañez Gómez, G. (1999). Elementos de historiografía de la geografía colombiana. Revista de Estudios Sociales, 3, 9-28.

Mora Pacheco, K. G. (2015). Monotonía, aislamiento y atraso agrícola. Descripciones de viajeros del siglo XIX e historia agraria de la Sabana de Bogotá (Colombia). HiSTOReLo. Revista de Historia Regional y Local, 7(14), 180-213. https://doi. org/10.15446/historelo.v7n14.48625.

Mörner, M. (1973). The Spanish American Hacienda: A Survey of Recent Research and Debate. The Hispanic American Historical Review, 53(2), 183-216. https://doi. org/10.2307/2512251.

Motta González, N. \& Perafán Cabrera, A. (2012). Historia ambiental del Valle del Cauca: geoespacialidad, cultura y género. Cali: Universidad del Valle.

Perafán Cabrera, A. (2012). Valle del Cauca: un estudio en torno a su sociedad y medio ambiente. Cali: Programa Editorial, Universidad del Valle.

Ramírez, M. C. (1984). La fragmentación de latifundios y el despegue municipal de Pradera: El caso del indiviso del Bolo de los Escobares. (Tesis de licenciatura). Universidad del Valle, Cali, Colombia.

Reyes Posada, A. (1978). Latifundio y poder político. La hacienda ganadera en Sucre. Bogotá: Editorial Cinep.

Rivera y Garrido, L. (1886). Algo sobre el Valle del Cauca (Impresiones y recuerdos de un conferencista). Buga: Imprenta R. A. Pastrana.

Valdivia Rojas, L. (1979). Descripción del mapa del Valle del río Cauca y sus afluentes. Historia y Espacio, 2, 117-127. doi: https://doi.org/10.25100/hye.v0i2.4717.

Valdivia Rojas, L. (1980). Mapas de densidad de población para el suroccidente 18431870. Historia y Espacio, 5(1), 102-110.

Valdivia Rojas, L. (1984). Origen y situación de la pequeña posesión campesina en el Valle del Cauca, siglo XIX. Historia y Espacio, 111(10), 56-110.

Valdivia Rojas, L. (1992). Economía y espacio: el Valle del Cauca 1850 a 1950. Cali: Universidad del Valle.

Valencia Llano, A. (2016). Afrodescendientes en el Valle del Cauca: Ensayos históricos. Cali: Universidad del Valle.

Van Ausdal, S. (2009a). Pasture, profit, and power: An environmental history of cattle ranching in Colombia, 1850-1950. Geoforum, 40(5), 707-719. https://doi. org/10.1016/j.geoforum.2008.09.012

Van Ausdal, S. (2009b). The logic of livestock: An historical geography of cattle ranching in Colombia, 1850-1950. (ProQuest Dissertations Publishing). Recuperado de http:// search.proquest.com/docview/506845301/

Velasco Arizabaleta, L. M. (1982). Historia del hábitat vallecaucano: 1536-1982. Cali: CVC. 